\title{
A GENERAL THEOREM ON THE CONVERGENCE OF OPERATOR SEMIGROUPS
}

\author{
BY \\ THOMAS G. KURTZ
}

1. Introduction. In a variety of contexts, a number of authors [2], [4]-[8] have considered the question of when a sequence of semigroups of linear operators

$$
T_{n}(t): L_{n} \rightarrow L_{n}
$$

converge to a semigroup of linear operators

$$
T(t): L \rightarrow L
$$

where the $L_{n}$ and $L$ are locally convex linear topological spaces.

Convergence of the $T_{n}(t)$ means that for a sequence $\left\{f_{n}\right\} f_{n} \in L_{n}$

$$
\lim _{n \rightarrow \infty} f_{n}=f \in L
$$

implies

$$
\lim _{n \rightarrow \infty} T_{n}(t) f_{n}=T(t) f
$$

In all of these theorems the notion of convergence used in (1-1) and (1-2) has essentially been strong convergence. It is the purpose of the present paper to prove analogous theorems for a certain class of notions of convergence, which will include, in the case of Banach spaces of functions with the sup norm, bounded, pointwise convergence, and convergence of bounded sequences which is uniform over compact sets.

To motivate the eventual abstract formulation of our problem, let us consider the setting, due to Trotter, which was used in [4] and [7].

Here the $L_{n}$ and $L$ are Banach spaces. We assume there exist continuous linear maps $P_{n}: L \rightarrow L_{n}$ such that

We say

$$
\lim _{n \rightarrow \infty}\left\|P_{n} f\right\|=\|f\| \quad \text { for every } f \in L .
$$

$$
\lim _{n \rightarrow \infty} f_{n}=f
$$

if and only if $\lim _{n \rightarrow \infty}\left\|f_{n}-P_{n} f\right\|=0$.

Presented to the Society, January 24, 1969 under the title $A$ general theorem on the convergence of sequences or semigroups of linear operators; received by the editors May 8, 1969.

Copyright (C) 1970, American Mathematical Society 
Let $\mathscr{L}$ be the Banach space of bounded sequences with

$$
\left\|\left\{f_{n}\right\}\right\|=\sup _{n}\left\|f_{n}\right\| .
$$

Let $\mathscr{D}(P) \subset \mathscr{L}$ be the space of convergent sequences and observe that

$$
P:\left\{f_{n}\right\} \in \mathscr{D}(P) \rightarrow \lim _{n \rightarrow \infty} f_{n} \in L
$$

defines a continuous linear operator from $\mathscr{D}(P)$ onto $L$.

This, then, will be our basic assumption: that the notion of convergence of a sequence $\left\{f_{n}\right\}$ defines a continuous linear operator $P$ from a subspace $\mathscr{D}(P)$ of the space of bounded sequences $\mathscr{L}$ to the limiting linear space $L$.

What is meant by a bounded sequence when the $L_{n}$ are different locally convex linear topological spaces (lclts) will be clear when we discuss the topology on $\mathscr{L}$. Seidman [6] considers nets instead of sequences, but it should be clear from the final formulation that this does not introduce any real complications.

If the $L_{n}$ are Banach spaces then (1-4) defines the primary topology $\tau$ on $\mathscr{L}$. If the $L_{n}$ are all the same sequentially complete lclts then we define a family of seminorms

$$
p\left(\left\{f_{n}\right\}\right)=\sup _{n} p\left(f_{n}\right)
$$

for every continuous seminorm $p$ on $L_{n}$, and these determine the topology $\tau$. If the $L_{n}$ are different lclts, let $\phi_{n}$ denote the collection of continuous seminorms on $L_{n}$ and let $\phi$ be some collection of sequences $\left\{p_{n}\right\}, p_{n} \in \phi_{n}$ such that $\left\{p_{n}: p_{n}\right.$ is in some sequence in $\phi\}$ determines the topology $\tau_{n}$ on $L_{n}$. Then for every sequence $\left\{p_{n}\right\} \in \phi$ we define a seminorm

$$
p\left(f_{n}\right)=\sup _{n} p_{n}\left(f_{n}\right)
$$

and these seminorms determine the topology $\tau$. We observe

implies

$$
\tau-\lim _{k \rightarrow \infty}\left\{f_{n}^{k}\right\}=\left\{f_{n}\right\}
$$

$$
\tau_{n}-\lim _{k \rightarrow \infty} f_{n}^{k}=f_{n}
$$

and $\mathscr{L}$ is sequentially complete in the topology $\tau$.

We now assume that $\mathscr{T}(t)\left\{f_{n}\right\}=\left\{T_{n}(t) f_{n}\right\}$ defines an equicontinuous semigroup on $\mathscr{L}$. This is the same as assuming some sort of mutual equicontinuity on the $T_{n}(t)$ and is implied by the assumptions made in the earlier papers, at least after multiplying each $T_{n}(t)$ by $e^{-K t}$ for some $K$ independent of $n$.

We observe that the statement: if $\left\{f_{n}\right\}$ is a convergent sequence then $\left\{T_{n}(t) f_{n}\right\}$ is a convergent sequence is the same as saying

$$
\mathscr{T}(t): \mathscr{D}(P) \rightarrow \mathscr{D}(P),
$$

and

$$
T(t) P\left\{f_{n}\right\}=P \mathscr{T}(t)\left\{f_{n}\right\}
$$


defines a semigroup on $L$ if in addition

$$
\mathscr{T}(t): \mathscr{N}(P) \rightarrow \mathscr{N}(P),
$$

where $\mathcal{N}(P)$ is the null space of $P$.

For the notion of convergence given by (1-3), (1-7) is trivial, but it may not be for other types of convergence satisfying our conditions.

We will assume for the moment that $T_{n}(t)$ is a $C_{0}$-semigroup on $\left(L_{n}, \tau_{n}\right)$, but we will see later that there are situations in which this assumption may be profitably altered.

Even under this assumption, $\mathscr{T}(t)$ is almost never a $C_{0}$-semigroup on $(\mathscr{L}, \tau)$. However, if we define a second topology $\sigma$ on $\mathscr{L}$ by seminorms of the form

$$
\phi\left(\left\{f_{n}\right\}\right)=\sum_{n=1}^{\infty} a_{n} p_{n}\left(f_{n}\right), \quad\left\{p_{n}\right\} \in \phi, \quad a_{n} \geqq 0 \quad \text { and } \sum_{n=1}^{\infty} a_{n}<\infty,
$$

$\mathscr{T}(t)$ is $\sigma$-continuous and every $\sigma$-bounded set is $\tau$-bounded. (In fact $\mathscr{L}$ is $\sigma$ sequentially complete, but this fact is not used in what follows.)

Let $A_{n}$ be the infinitesimal operator of $T_{n}(t)$ and define $\mathscr{A}\left\{f_{n}\right\}=\left\{A_{n} f_{n}\right\}$ for every $\left\{f_{n}\right\}$ such that $f_{n} \in \mathscr{D}\left(A_{n}\right)$ and $\left\{A_{n} f_{n}\right\} \in \mathscr{L}$. We observe that $(\lambda-\mathscr{A})^{-1}$ is defined on $\mathscr{L}$ and is given by the $\sigma$-Riemann improper integral

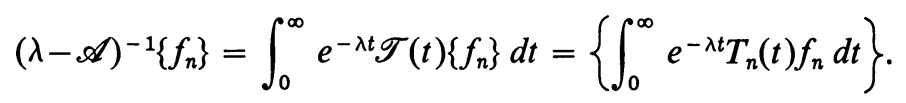

The subspace $\mathscr{L}_{0} \subset \mathscr{L}$ on which $\mathscr{T}(t)$ is a $C_{0}$-semigroup in $\tau$ is just the closure of $\mathscr{D}(\mathscr{A})$, the domain of $\mathscr{A}$.

The main problem is to find conditions under which (1-5) holds. To do this we will exploit the following corollary to the Hille-Yosida Theorem.

Proposition (1-8). Let $\mathscr{L}_{0}$ be a lclts with topology $\tau$ and suppose $\mathscr{T}(t)$ is a $C_{0}$ $\tau$-equicontinuous semigroup of linear operators on $\mathscr{L}_{0}$ and that $\mathscr{A}_{0}$ denotes its infinitesimal operator. If $D$ is a closed linear subspace of $\mathscr{L}_{0}$ then

implies

$$
\left(\lambda-\mathscr{A}_{0}\right)^{-1}: D \rightarrow D \text { for some } \lambda>0
$$

$$
\mathscr{T}(t): D \rightarrow D \text { all } t \geqq 0 .
$$

We will now restate our problem in a slightly more general context which will suppress the sequential nature of $\mathscr{L}$.

Let $\mathscr{L}$ and $L$ be lclts, and let $P$ be a continuous linear operator from a closed subspace $\mathscr{D}(P) \subset \mathscr{L}$ onto $L$. When does there exist a subspace $D \subset \mathscr{D}(P)$ such that

$$
T(t) P f=P \mathscr{T}(t) f, \quad f \in D
$$

defines a semigroup on the subspace $P(D) \subset L$ ?

This is the form in which the problem is considered in $\$ 2$.

2. Transformation of a semigroup. The following assumptions are all satisfied by the corresponding objects considered in the introduction. 
Let $\mathscr{L}$ be a linear space and let $\tau$ and $\sigma$ be locally convex topologies on $\mathscr{L}$ satisfying

(2-1) $\sigma \subset \tau$;

(2-2) a set $\Gamma \subset \mathscr{L}$ is $\sigma$-bounded if and only if it is $\tau$-bounded;

(2-3) $\mathscr{L}$ is $\tau$-sequentially complete.

Let $\mathscr{T}(t): \mathscr{L} \rightarrow \mathscr{L}$ be a semigroup of linear operators satisfying

(2-4) $\mathscr{T}(t)$ is $\tau$-equicontinuous;

(2-5) $\sigma-\lim _{t \rightarrow 0} \mathscr{T}(t) f=f$ for every $f \in \mathscr{L}$;

(2-6) $\sigma-\lim _{n \rightarrow \infty} f_{n}=f$ implies $\sigma-\lim _{n \rightarrow \infty} \mathscr{T}(t) f_{n}=\mathscr{T}(t) f$;

(2-7) $\int_{a}^{b} \mathscr{T}(t) f d t$ exists in $\mathscr{L}$ for every $b>a>0$ and $f \in \mathscr{L}$ as a $\sigma$-Riemann integral;

(2-8) $\int_{0}^{\infty} e^{-\lambda t} \mathscr{T}(t) f d t$ exists in $\mathscr{L}$ for every $\lambda>0$ and $f \in \mathscr{L}$, as an improper $\sigma$-Riemann integral.

Define

$$
\mathscr{A} f=\sigma-\lim _{t \rightarrow 0} \frac{\mathscr{T}(t) f-f}{t}
$$

and

$$
\mathscr{A}_{0} f=\tau-\lim _{t \rightarrow 0} \frac{\mathscr{T}(t) f-f}{t}
$$

when these limits exist in $\mathscr{L}$. Clearly $\mathscr{A}$ is an extension of $\mathscr{A}_{0}$. It follows that

$$
(\lambda-\mathscr{A})^{-1} f=\int_{0}^{\infty} e^{-\lambda t} \mathscr{T}(t) f d t \quad \text { for every } f \in \mathscr{L},
$$

and $\left(\lambda-\mathscr{A}_{0}\right)^{-1}$ is given by the same formula but is defined only on the subspace $\mathscr{L}_{0} \subset \mathscr{L}$ on which $\mathscr{T}(t)$ is a $C_{0}$-semigroup in $\tau$.

From the Hille-Yosida Theorem, (2-2) and the definition of $\mathscr{A}$, it follows that $\mathscr{L}_{0}$ is the $\tau$-closure of both $\mathscr{D}(\mathscr{A})$ and $\mathscr{D}\left(\mathscr{A}_{0}\right)$.

We note in passing that Komatsu [3] has considered what he calls $(\tau, \sigma)$ semigroups under assumptions closely related to ours.

From here on, all statements involving a topology on $\mathscr{L}$ will be understood to mean $\tau$ unless explicitly stated otherwise.

Let $L$ be another lclts, $\mathscr{D}(P) \subset \mathscr{L}$ a $\tau$-closed subspace and $P$ a $\tau$-continuous linear operator from $\mathscr{D}(P)$ onto $L$ with null space $\mathscr{N}(P)$.

The space $L$ is said to have the induced topology or the topology induced by $P$ if the topology is given by $\left\{v: P^{-1}(v) \in \tau\right\}$. The following lemma collects some conditions under which $L$ has the induced topology.

Lemma (2-9). Denoting the closure of $a$ set $B$ by $\bar{B}$, the following are equivalent:

(i) L has the induced topology;

(ii) $\bar{B}=P \overline{\left(P^{-1}(B)\right)}$ for every $B \subset L$;

(iii) if $q$ is a continuous seminorm on $\mathscr{L}$ then

$$
\tilde{q}(g)=\inf \{q(f): P f=g\}
$$


defines a continuous seminorm on $L$ and the topology on $L$ is determined by seminorms of this form.

In particular $L$ has the induced topology if there exists a continuous linear operator $Q: L \rightarrow \mathscr{D}(P)$ such that $P Q f=f$ for every $f \in L$.

The following is a consequence of Proposition (1-8).

Proposition (2-10). Suppose

$$
\mathscr{T}(t): \mathscr{N}(P) \rightarrow \mathscr{N}(P)
$$

and for some $\lambda>0$

$$
(\lambda-\mathscr{A})^{-1}: \mathscr{D}(P) \rightarrow \mathscr{D}(P)
$$

Then

and hence by (2-11)

$$
\mathscr{T}(t): \mathscr{D}(P) \cap \mathscr{L}_{0} \rightarrow \mathscr{D}(P) \cap \mathscr{L}_{0}
$$

$$
\mathscr{T}(t): \overline{P^{-1}\left(P\left(\mathscr{D}(P) \cap \mathscr{L}_{0}\right)\right)} \equiv \Gamma \rightarrow \Gamma,
$$

and $T(t) P f=P \mathscr{T}(t) f, f \in \Gamma$ defines a semigroup of linear operators on $P(\Gamma) \subset L$.

Finally we have the main theorem.

THEOREM (2-13). Suppose

$$
\mathscr{T}(t): \mathscr{N}(P) \rightarrow \mathscr{N}(P)
$$

and for some $\lambda>0$

$$
(\lambda-\mathscr{A})^{-1}: \mathscr{N}(P) \rightarrow \mathscr{N}(P) .
$$

We define a possibly multivalued operator $A: \mathscr{D}(A) \subset L \rightarrow L$ by

$$
\begin{aligned}
A g & =\{P \mathscr{A} f: f, \mathscr{A} f \in \mathscr{D}(P) \text { and } P f=g\}, \\
\mathscr{D}(A) & =\{g: A g \neq \varnothing\} .
\end{aligned}
$$

Then there exists $\lambda>0$ such that the range $\mathscr{R}(\lambda-A)$ of $\lambda-A$ satisfies

$$
\mathscr{R}(\lambda-A)=L
$$

if and only if there exists $\lambda>0$ such that

$$
(\lambda-\mathscr{A})^{-1}: \mathscr{D}(P) \rightarrow \mathscr{D}(P) .
$$

Under these conditions

$$
\mathscr{T}(t): \Gamma \rightarrow \Gamma
$$

and $T(t) P f=P \mathscr{T}(t) f, f \in \Gamma$ defines a semigroup of linear operators on $P(\Gamma)$.

Assuming that $L$ has the induced topology, $\mathscr{R}(\lambda-A)$ is always closed and hence (2-16) may be replaced by

$$
\mathscr{R}(\lambda-A) \text { is dense in } L \text {. }
$$


A further consequence of $L$ having the induced topology is that $\left\{\lambda^{n}(\lambda-A)^{-n}\right\}$ form an equicontinuous family of operators. If (2-16) or (2-17) hold, $P(\Gamma)$ is the closure of $P\left(\mathscr{D}(P) \cap \mathscr{L}_{0}\right)$ which is the same as the closure of

$$
\mathscr{D}(A) \equiv P\left((\lambda-A)^{-1} \mathscr{D}(P)\right) \text {. }
$$

It follows from the Hille-Yosida Theorem that $T(t)$ is a $C_{0}$-semigroup of equicontinuous linear operators on $P(\Gamma)$ and the infinitesimal operator $A_{0}$ is the restriction of $A$ to

$$
\mathscr{D}\left(A_{0}\right)=\{f \in \mathscr{D}(A): A f \in P(\Gamma)\} .
$$

Proof. Let $D=\{g: g=\lambda f-\mathscr{A} f, f, \mathscr{A} f \in \mathscr{D}(P)\}$. Then $\mathscr{R}(\lambda-A)=P(D)$ and since (2-17) implies $D=\mathscr{D}(P)(2-16)$ follows.

Conversely, (2-15) implies

$$
D=P^{-1}(P(D))
$$

and hence (2-16) implies $D=P^{-1}(L)=\mathscr{D}(P)$. Since $(\lambda-\mathscr{A})^{-1}: D \rightarrow \mathscr{D}(P),(2-17)$ follows.

Assume now that $L$ has the induced topology. We want to show $\mathscr{R}(\lambda-A)$ is closed. By Lemma (2-9)

$$
\begin{aligned}
\overline{\mathscr{R}(\lambda-A)} & =P \overline{\left(P^{-1}(\mathscr{R}(\lambda-A))\right)}=P \overline{\left(P^{-1}(P(D))\right)} \\
& =P(\bar{D})=P(D)=\mathscr{R}(\lambda-A) .
\end{aligned}
$$

The fact that $\left\{\lambda^{n}(\lambda-A)^{-n}\right\}$ form an equicontinuous family follows from Lemma (2-9) part (iii) and the fact that $\left\{\lambda^{n}(\lambda-\mathscr{A})^{-n}\right\}$ form an equicontinuous family on $\mathscr{L}$. The rest is clear.

It is natural to ask what can be said if $f \in \mathscr{D}(P)$ but $f \notin \Gamma$. We observe that (2-6), (2-7) and (2-8) imply for every $f \in \mathscr{L}$

$$
\begin{aligned}
\mathscr{T}(s) \int_{a}^{b} \mathscr{T}(t) f d t & =\int_{a}^{b} \mathscr{T}(t+s) f d t \\
\mathscr{T}(s) \int_{0}^{\infty} e^{-\lambda t} \mathscr{T}(t) f d t & =\int_{0}^{\infty} e^{-\lambda t} \mathscr{T}(t+s) f d t
\end{aligned}
$$

and

$$
h=\int_{a}^{b} \mathscr{T}(t) f d t \in \mathscr{D}(\mathscr{A})
$$

with $\mathscr{A} h=\mathscr{T}(b) f-\mathscr{T}(a) f$. Under the conditions of Theorem (2-13)

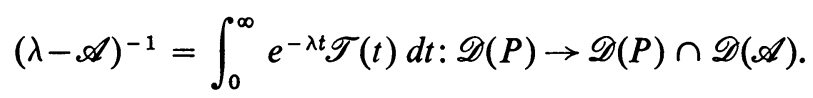

Therefore for $f \in \mathscr{D}(P)$

$$
\int_{0}^{\infty} e^{-\lambda t \mathscr{T}}(t) f d t \in \mathscr{D}(P) \cap \mathscr{D}(\mathscr{A})
$$


and

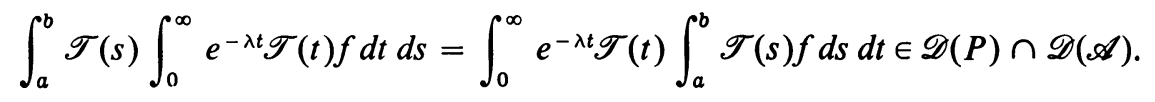

Since $\int_{a}^{b} \mathscr{T}(s) f d t \in \mathscr{L}_{0}$

$$
\tau-\lim _{\lambda \rightarrow \infty} \lambda \int_{0}^{\infty} e^{-\lambda t} \mathscr{T}(t) \int_{a}^{b} \mathscr{T}(s) f d s d t=\int_{a}^{b} \mathscr{T}(s) f d s
$$

and since $\mathscr{D}(P) \cap \mathscr{L}_{0}$ is $\tau$-closed

$$
\int_{a}^{b} \mathscr{T}(s) f d s \in \mathscr{D}(P) \cap \mathscr{L}_{0}
$$

Rewriting this result in a more suggestive fashion we have

Proposition (2-22). Under the conditions of Theorem (2-13), for every $f \in \mathscr{D}(P)$, $t \geqq 0$ and $\varepsilon>0$

$$
\mathscr{T}(t) \frac{1}{\varepsilon} \int_{0}^{\varepsilon} \mathscr{T}(s) f d s=\frac{1}{\varepsilon} \int_{0}^{\varepsilon} \mathscr{T}(t+s) f d s \in \mathscr{D}(P) \cap \mathscr{L}_{0}
$$

In terms of sequences, if $\lim _{n \rightarrow \infty} f_{n}$ exists, then

$$
\lim _{n \rightarrow \infty} \frac{1}{\varepsilon} \int_{0}^{\varepsilon} T_{n}(t+s) f_{n} d s
$$

exists.

3. Relation to other theorems. In the earlier work on semigroup convergence theorems, the last condition in Lemma (2-9) is always satisfied; consequently the assumption that $L$ has the induced topology holds. As was pointed out earlier, convergence in these papers is always essentially strong convergence so (2-14) and (2-15) are trivially satisfied. Seidman and Kato assume that the resolvents converge which corresponds to (2-17). The assumptions used by Trotter and in the earlier work of this author imply $\left(2-16^{\prime}\right)$. The additional assumption that either the range of the limiting resolvent or $\mathscr{D}(A)$ is dense in $L$ serves only to imply that $P(\Gamma)=L$.

As a further application of Theorem (2-13) consider a sequence of contraction semigroups defined on Banach spaces $S_{n}(t): K_{n} \rightarrow K_{n}$, and let $T_{n}(t)$ be the dual of $S_{n}(t)$ restricted to some Banach subspace $L_{n} \subset K_{n}^{*}$, such that $T_{n}(t): L_{n} \rightarrow L_{n}$.

Let $\sigma_{n}$ denote the weak* topology on $L_{n}$ and $\tau_{n}$ the norm topology. Assume $\sigma_{n}-\lim _{t \rightarrow 0} T_{n}(t) f=f$ for every $f \in L_{n}$ and that $L_{n}$ is closed under $\sigma_{n}$-Riemann integration of $\sigma_{n}$-right continuous functions. That is $u(t) \in L_{n}, a \leqq t \leqq b$ and $u(t) \sigma_{n}$-right continuous implies

Define

$$
\int_{a}^{b} u(t) d t \in L_{n}
$$

$$
\tilde{A}_{n} f=\sigma_{n}-\lim _{t \rightarrow 0} \frac{T_{n}(t) f-f}{t}
$$


when the limit exists in $L_{n}$. It can be shown, Dynkin [1], that $\tilde{A}_{n}$ is a $\tau_{n}$-closed operator and that

$$
\left(\lambda-\tilde{A}_{n}\right)^{-1} f=\int_{0}^{\infty} e^{-\lambda t} T_{n}(t) f d t, \quad \lambda>0
$$

Let $\mathscr{L}$ be the Banach space of bounded sequences, $\tau$, as before, the topology given by $\left\|\left\{f_{n}\right\}\right\|=\sup _{n}\left\|f_{n}\right\|$ and $\sigma$ the topology given by seminorms of the form

$$
q\left(\left\{f_{n}\right\}\right)=\sum_{n=1}^{\infty}\left|\nu_{n}\left(f_{n}\right)\right|
$$

where $\nu_{n} \in K_{n}$ and $\sum_{n=1}^{\infty}\left\|\nu_{n}\right\|<\infty$. Define $\mathscr{T}(t)\left\{f_{n}\right\}=\left\{T_{n}(t) f_{n}\right\}$. Then $\mathscr{L}, \tau, \sigma$ and $\mathscr{T}(t)$ satisfy conditions (2-1) through (2-8) and $\mathscr{A}$ is given by $\mathscr{A}\left\{f_{n}\right\}=\left\{\tilde{A}_{n} f_{n}\right\}$ with

$$
\mathscr{D}(\mathscr{A})=\left\{\left\{f_{n}\right\} \in \mathscr{L}:\left\{\tilde{A}_{n} f_{n}\right\} \in \mathscr{L}\right\} .
$$

We therefore have limit theorems in which the limiting infinitesimal operator $A$ is the limit of the sequence of weak infinitesimal operators.

4. The discrete parameter case. Let $T_{n}: L_{n} \rightarrow L_{n}$ be a sequence of continuous linear operators and let $\mathscr{L}, \tau$ and $\sigma$ be defined as in $\S 1$. Let $T_{n}\{k$ ? denote the $k$ th power of the operator $T_{n}$ and suppose the collection of operators $\left\{\mathscr{S}\left(\left\{k_{n}\right\}\right)\right\}$ defined by

$$
\mathscr{S}\left(\left\{k_{n}\right\}\right)\left\{f_{n}\right\}=\left\{T_{n}\left(k_{n}\right) f_{n}\right\},
$$

$\left\{k_{n}\right\}$ a sequence of nonnegative integers, is an equicontinuous family of operators on $\mathscr{L}$.

Let $h_{n}>0$ satisfy $\lim _{n \rightarrow \infty} h_{n}=0$ and define $A=\left(T_{n}-I\right) / h_{n}$. Then

$$
\mathscr{T}(t)\left\{f_{n}\right\}=\left\{\exp \left\{t A_{n}\right\} f_{n}\right\}
$$

defines a semigroup on $\mathscr{L}$ satisfying conditions (2-4) through (2-8) and $\mathscr{A}\left\{f_{n}\right\}$ $\left\{A_{n} f_{n}\right\}$ with

$$
\mathscr{D}(\mathscr{A})=\left\{\left\{f_{n}\right\} \in \mathscr{L}:\left\{A_{n} f_{n}\right\} \in \mathscr{L}\right\} .
$$

Let $P$ be a continuous linear operator $P: \mathscr{D}(P) \subset \mathscr{L} \rightarrow L$, and suppose $\left\{f_{n}\right\} \in \mathscr{L}$ implies $\left\{h_{n} f_{n}\right\} \in \mathscr{N}(P)$. Then

$$
\left(\mathscr{T}(t)-\mathscr{S}\left(\left\{\left[t / h_{n}\right]\right\}\right)\right): \mathscr{L}_{0} \rightarrow \mathscr{N}(P) .
$$

The proof of this is essentially the same as the proof of Lemma (2-19) in [4] and it reduces the proof of the convergence of a sequence of discrete parameter semigroups to the proof of the convergence of a sequence of continuous parameter semigroups.

5. Hasegawa's conditions. One other type of condition for the convergence of sequences of semigroups has been investigated by M. Hasegawa [2] and S. Oharu [5]. 
In the Banach space case, with all the semigroups defined on the same space $L$ and strong convergence, this condition is

$$
\lim _{n, m \rightarrow \infty}\left\|T_{n}(t) T_{m}\left(t^{\prime}\right) f-T_{m}\left(t^{\prime}\right) T_{n}(t) f\right\|=0 \quad \text { for all } t, t^{\prime} \geqq 0 \text { and all } f \in L .
$$

In order to restate this condition in the context of $\$ 2$ we observe that a sequence $\left\{f_{n}\right\} \subset L$ is a Cauchy sequence if and only if

$$
\lim _{n \rightarrow \infty} f_{n}-f_{\pi_{n}}=0
$$

for every permutation $\left\{\pi_{n}\right\}$ of the positive integers, and that $H\left\{f_{n}\right\}=\left\{f_{\pi_{n}}\right\}$ defines a continuous linear operator on $\mathscr{L}$ which has a continuous inverse.

With this in mind and with $\mathscr{L}, \tau, \sigma, P$ etc. as in $\S 2$, we assume that $\mathscr{H}$ is a collection of $\sigma$-continuous linear operators on $\mathscr{L}$ such that $H \in \mathscr{H}$ implies $H^{-1} \in \mathscr{H}$ and $f \in \mathscr{D}(P)$ if and only if

$$
H f-f \in \mathscr{N}(P) \text { for every } H \in \mathscr{H} \text {. }
$$

Since $\mathscr{N}(P)$ is a linear subspace it follows that $H: \mathscr{N}(P) \rightarrow \mathscr{N}(P)$.

In this context condition (5-1) can be stated as

$$
\begin{aligned}
\mathscr{T}(t) H^{-1} \mathscr{T}\left(t^{\prime}\right) H-H^{-1} \mathscr{T}\left(t^{\prime}\right) H \mathscr{T}(t): & \mathscr{D}(P) \rightarrow \mathscr{N}(P) \\
& \text { for all } t, t^{\prime} \geqq 0 \text { and every } H \in \mathscr{H} .
\end{aligned}
$$

Defining

$$
R_{\lambda}=(\lambda-\mathscr{A})^{-1}=\int_{0}^{\infty} e^{-\lambda t} \mathscr{T}(t) d t
$$

a variety of assumptions about the nature of the limiting process would allow us to conclude from (5-2) that

$$
R_{\lambda} H^{-1} R_{\lambda} H-H^{-1} R_{\lambda} H R_{\lambda}: \mathscr{D}(P) \rightarrow \mathscr{N}(P), \quad \text { every } H \in \mathscr{H} \text { and some } \lambda>0,
$$

but rather than make additional assumptions about $P$ we shall take $\left(5-2^{\prime}\right)$ as the basic condition.

Let

$$
\mathscr{D}_{c}=\{f \in \mathscr{D}(\mathscr{A}) \cap \mathscr{D}(P): \mathscr{A} f \in \mathscr{D}(P)\}
$$

We will show, assuming condition (5-2') and

$$
R_{\lambda}: \mathscr{N}(P) \rightarrow \mathscr{N}(P) \text { for every } \lambda>0,
$$

that

$$
R_{\lambda}: \mathscr{D}_{c} \rightarrow \mathscr{D}(P)
$$

and hence, since $R_{\lambda}\left(\mathscr{D}_{c}\right) \cap \mathscr{D}(P) \subset \mathscr{D}_{c}$

$$
R_{\lambda}: \overline{P^{-1}\left(P\left(\overline{\mathscr{D}}_{c}\right)\right)} \equiv \tilde{\Gamma} \rightarrow \tilde{\Gamma} .
$$


Since $\overline{\mathscr{D}}_{c}$ is a closed subspace of $\mathscr{L}_{0} \cap \mathscr{D}(P)$, Proposition (1-8) implies the following:

TheOREM (5-6). Let $\mathscr{H}, \mathscr{D}_{c}$ and $\tilde{\Gamma}$ be defined as above. Then (5-2'), (5-3) and

$$
\mathscr{T}(t): \mathscr{N}(P) \rightarrow \mathscr{N}(P)
$$

imply

$$
T(t) P f=P \mathscr{T}(t) f, \quad f \in \tilde{\Gamma},
$$

defines a semigroup on $P(\tilde{\Gamma})$. If $L$ has the induced topology and $A$ is defined as in Theorem (2-13), then $T(t)$ is strongly continuous on $P(\tilde{\Gamma})$ which is the closure of $\mathscr{D}(A)$.

To verify (5-4) we need to show that for every $f \in \mathscr{D}_{c}$ and every $H \in \mathscr{H}$

$$
H R_{\lambda} f-R_{\lambda} f \in \mathscr{N}(P) \text {. }
$$

Using (5-3), (5-2') and the fact that $H-I: \mathscr{D}(P) \rightarrow \mathscr{N}(P)$ we have

$$
\begin{aligned}
H R_{\lambda} f-R_{\lambda} f= & H R_{\lambda}\left(I-H^{-1}\right) f+R_{\lambda}(H-I) f+H R_{\lambda} H^{-1} f-R_{\lambda} H f \\
= & H R_{\lambda}\left(I-H^{-1}\right) f+R_{\lambda}(H-I) f+H R_{\lambda} H^{-1} R_{\lambda}(\lambda-A) f \\
& -R_{\lambda} H R_{\lambda} H^{-1}(\lambda-A) f-R_{\lambda} H R_{\lambda}\left(I-H^{-1}\right)(\lambda-A) f \in \mathscr{N}(P),
\end{aligned}
$$

since $f \in \mathscr{D}_{c}$ implies $(\lambda-A) f \in \mathscr{D}(P)$.

REMARK. Many applications of the earlier semigroup approximation theorems have been found for sequences of Markov processes. This area, in fact, motivated the present work. The Banach spaces involved in Markov process applications are usually spaces of functions with the sup norm. The type of convergence that arises naturally is bounded, pointwise convergence which, as was pointed out in the introduction, fits into the context of this paper.

\section{REFERENCES}

1. E. B. Dynkin, Markov processes, Vol. I, Fizmatgiz, Moscow, 1963; English transl., Academic Press, New York and Springer-Verlag, Berlin, 1965. MR 33 \#1886; MR 33 \#1887.

2. M. Hasegawa, A note on the convergence of semi-groups of operators, Proc. Japan Acad. 40 (1964), 262-266. MR 30 \#3376.

3. H. Komatsu, Semi-groups of operators in locally convex spaces, J. Math. Soc. Japan 16 (1964), 230-262. MR 32 \#368.

4. T. G. Kurtz, Extensions of Trotter's operator semigroup approximation theorems, J. Functional Analysis 3 (1969), 111-132.

5. S. Ôharu, On the convergence of semi-groups of operators, Proc. Japan Acad. 42 (1966), 880-884. MR 35 \#3481.

6. T. Seidman, Approximation of operator semigroups, Department of Mathematics, Carnegie Institute of Technology, Report 67-34.

7. H. F. Trotter, Approximation of semi-groups of operators, Pacific J. Math. 8 (1958), 887-919. MR 21 \#2190.

8. K. Yosida, Functional analysis, Academic Press, New York and Springer-Verlag, Berlin, 1965. Section IX 12, theorems attributed to T. Kato. MR 31 \#5054.

UNIVERSITY OF WISCONSIN, MADISON, WisCONSIN 